\title{
Comparison of postoperative pain according to the harvesting method used in hair restorative surgery
}

\author{
Yang Seok Kim ${ }^{1}$, Young Cheon $\mathrm{Na}^{1}$, Jae Hyun Park ${ }^{2}$ \\ ${ }^{1}$ Department of Plastic and Reconstructive Surgery, Wonkwang University School of Medicine, Iksan; ${ }^{2}$ Dana Plastic Surgery Clinic, Seoul, \\ Korea
}

Background Postoperative pain is one of the most common concerns of patients undergoing hair transplantation surgery. Because most patients are satisfied with the cosmetic improvement after transplantation, amelioration of postoperative pain would help to increase patient accessibility to hair restorative surgery and greatly impact patient satisfaction with the final cosmetic results. This study was performed to investigate postoperative pain after hair transplantation.

Methods In total, 241 patients (202 who underwent follicular unit transplantation [FUT] and 39 who underwent follicular unit extraction [FUE]) were eligible for the study. Postoperative pain was evaluated on postoperative days 1, 2, 3, 4, 5, and 7 using the Wong-Baker Faces Pain Scale. The patients' medical records were retrospectively reviewed for information on the harvesting method, number of transplanted grafts, size of donor design, and laxity, elasticity, and glidability of the scalp in relation to postoperative pain.

Results Postoperative pain after hair transplantation, assessed with the Wong-Baker Faces Pain Scale, seemed to provide very subjective results. None of the variables were correlated with postoperative pain in the FUT group. Such pain, however, tended to disappear by postoperative day 3. Patients in the FUE group experienced significantly less severe pain than those in the FUT group.

Conclusions Postoperative pain was significantly less severe in patients whose donor hair was harvested by the FUE than FUT method. Postoperative pain had almost disappeared by postoperative day 3 in the FUT group, whereas only minimal pain was present even on postoperative day 1 in the FUE group.

Keywords Pain / Pain measurement / Pain management / Hair follicle

Received: 26 Jun 2018 • Revised: 22 Jan $2019 \bullet$ Accepted: 26 Jan 2019

pISSN: 2234-6163 • elSSN: 2234-6171 • https://doi.org/10.5999/aps.2018.00843 • Arch Plast Surg 2019;46:241-247
Correspondence: Jae Hyun Park Dana Plastic Surgery Clinic, 606 Gangnam-daero, Gangnam-gu, Seoul 06038, Korea

Tel: +82-2-512-0922

Fax: +82-2-512-0942

E-mail: jay8384@naver.com

\section{INTRODUCTION}

Postoperative pain can have a significant effect on patients' recovery. A proper understanding of patients' attitudes and concerns about postoperative pain is an essential part of postoperative patient care [1] because the anticipation of such pain is known to cause high anxiety in patients with an impending surgical procedure. Many patients with alopecia who are planning to undergo hair transplantation are also concerned about postoperative pain [2]. Information encountered on the internet or obtained from an acquaintance who has experienced extreme pain increases fear in these patients, and they may consequently 
decline to undergo surgery. Most hair surgeons have adopted various methods in an endeavor to lessen intraoperative pain using intravenous anesthesia, ice packing, or vibrators [3]. Bupivacaine can be applied or analgesics can be prescribed for postoperative pain [4]. However, despite such efforts, the degree of postoperative pain varies greatly from no apparent pain to excruciating pain.

To the author's knowledge, no studies have been performed to investigate the relationship between postoperative pain and various factors such as the harvesting method (follicular unit extraction [FUE] vs. follicular unit transplantation [FUT]); number of grafts; size of surgical design; laxity, elasticity, and glidability of the scalp; and sex. Additionally, pain characteristics and duration have not been studied. Therefore, the present study was performed to investigate such details and to report the results with a review of the literature.

\section{METHODS}

This study was approved by the Internal Institutional Review Board of the Korea National Institute of Bioethics Policy (IRB No. 2015-0144-001). In total, 241 patients who underwent hair transplant surgery ( 202 by FUT and 39 by FUE) from June 2015 to June 2016 were eligible for the study. The operations were performed for male pattern baldness $(n=129)$, cicatricial alopecia of the scalp $(n=8)$, hairline correction $(n=103)$, and pubic hair restoration $(n=1)$.

In the FUT method, a long elliptical-shaped strip is removed from the donor area, followed by wound approximation at the margins to be closed with sutures. In the FUE method, individual follicular units are extracted after incising them with a punch 0.8-1.0 mm diameter in width.

Patients with a history of hair transplantation, laser treatment before or after surgery, eyelid surgery, rhinoplasty, filler injection, or any other type of surgery or procedures; those undergoing combined FUT and FUE surgery; and those who did not take the prescribed medication (oral nonsteroidal anti-inflammatory drugs or antibiotics) for various reasons such as adverse effects to drugs were excluded from the study to ensure a precise comparison.

Local anesthesia (2\% lidocaine with 1:100,000 epinephrine) was applied under intravenous sedation. Written informed consent for participation in the study was received from all patients prior to surgery for this prospective study. Postoperative pain assessment was performed on a daily basis.

Laxity, elasticity, and glidability of the scalp were accurately measured in all patients undergoing FUT. Laxity was measured with Dr. Parsa Mohebi Laxometer II. Elasticity was measured using Park's method [5], in which glidability was calculated by subtracting the elasticity value from the laxity value.

After surgery, the patients were routinely prescribed oral nonsteroidal anti-inflammatory medications (aceclofenac) for 3 days.

\section{Pain assessment}

Postoperative pain was assessed on postoperative days 1, 2, 3, 4, 5 , and 7 using the 5-point Wong-Baker Faces Pain Scale: 0 point, no hurt; 1 point, Hurts a little bit; 2 points: hurts a little bit more; 3 points: hurts even more; 4 points: hurts a whole lot; 5 points, hurts worst [6].

Postoperative pain was assessed on a daily basis until it reached 0 point on the Wong-Baker Faces Pain Scale. Data on the surgical method, donor design, and laxity, elasticity, and glidability of the scalp were retrospectively collected from the patients' medical records to evaluate the relationship of these factors with postoperative pain. The degree of pain according to purpose of undergoing surgery (alopecia, hairline correction, etc.) was not analyzed.

\section{Statistical analysis}

The results of the study were analyzed using SPSS for Windows version 21.0 (IBM Corp., Armonk, NY, USA).

\section{Follicular unit transplantation}

Pearson correlation coefficients were used to analyze the relationships between variables. One-way analysis of variance (ANOVA) was conducted to evaluate the difference in the degree of postoperative pain according to the duration after surgery. Repeated-measures ANOVA was applied to evaluate difference in postoperative pain level (Wong-Baker Scale) between men and women.

\section{Follicular unit extraction}

One-way ANOVA was used to evaluate the difference in postoperative pain (Wong-Baker Scale) according to various durations after surgery. Scheffe post hoc analysis was applied to detect the source of significance among groups that showed significant differences. Differences in postoperative pain (Wong-Baker Scale) according to the time that had elapsed after surgery were analyzed with repeated-measures ANOVA.

\section{FUT versus FUE}

The independent-sample t-test was employed to evaluate the significance of differences in postoperative pain between the FUT and FUE groups. 


\section{RESULTS}

\section{Follicular unit transplantation Correlation analysis}

Pearson correlation analysis was performed to assess the associations between all relevant variables. Age was positively correlated with the number of transplanted grafts $(\mathrm{r}=0.271, \mathrm{P}<$ $0.001)$, strip width $(\mathrm{r}=0.203, \mathrm{P}<0.01)$, and strip length $(\mathrm{r}=$ $0.295, \mathrm{P}<0.001)$ and negatively correlated with scalp laxity $(\mathrm{r}=$ $-0.170, \mathrm{P}<0.05)$. Additionally, the number of harvested grafts had a linear relationship with strip width $(\mathrm{r}=0.891, \mathrm{P}<0.001)$, strip length $(r=0.706, P<0.001)$, scalp elasticity $(r=0.382, P<$ $0.001)$, and scalp glidability $(\mathrm{r}=0.402, \mathrm{P}<0.001)$.

Strip width showed a positive correlation with strip length $(\mathrm{r}=0.532, \mathrm{P}<0.001)$, scalp laxity $(\mathrm{r}=0.487, \mathrm{P}<0.001)$, and scalp glidability $(\mathrm{r}=0.496, \mathrm{P}<0.001)$. Strip length was positively correlated with scalp glidability $(\mathrm{r}=0.165, \mathrm{P}<0.05)$. Scalp elasticity was positively correlated with scalp laxity $(\mathrm{r}=0.510$, $\mathrm{P}<0.001)$ and scalp glidability $(\mathrm{r}=0.764, \mathrm{P}<0.001)$.

Men tended to require larger amounts of donor hair, therefore required wider and longer donor designs. Men had higher laxity than women. Statistically significant differences in the pain score between men and women were found on postoperative days $1,2,3,4,5$, and 7 (Table 1 ).

\section{Degree of postoperative pain by day}

In the pain assessment using the Wong-Baker Faces Pain Scale, postoperative pain in those who underwent hair transplantation by the FUT method was rated at $2.03,1.47,0.91,0.56,0.36$, and 0.21 points on days $1,2,3,4,5$, and 7 , respectively.

On the first day after transplantation, $9.5 \%$ of patients experienced extreme pain ( $\geq 4$ points); this dramatically decreased to $2.0 \%$ of patients on the 2 nd day and to $0.5 \%$ on the 3 rd day. On the 3 rd day, $91.5 \%$ of patients reported a pain score of 0 to 2 , with only $8.0 \%$ of patients experiencing pain of 3 points. The postoperative pain was almost completely relieved or had disappeared by the 3rd day (Table 2).

Post hoc analysis to identify significant between-group differences showed that pain on the first day was significantly greater than pain on postoperative days 2, 3, 4, 5, and 7. Pain on postoperative day 2 was likewise significantly greater than that on days $3,4,5$, and 7 ; pain on day 3 was significantly greater than that on days 4,5 , and 7 ; and pain on day 4 was significantly greater than that on day 7 (Table 3 ).

\section{Relationship between postoperative pain and various factors}

The analysis of the relationship between postoperative pain and each variable showed no statistically significant relationship with age, number of grafts, width and length of surgical design for do-

Table 1. Difference between variables by sex

\begin{tabular}{|c|c|c|c|c|}
\hline \multirow{2}{*}{ Variable } & \multicolumn{2}{|c|}{ Sex } & \multirow{2}{*}{ t-value } & \multirow{2}{*}{ P-value } \\
\hline & Male $(n=109)$ & Female $(n=93)$ & & \\
\hline Age (yr) & $32.9 \pm 7.94$ & $30.6 \pm 6.59$ & 2.203 & $0.029^{*}$ \\
\hline No. of transplanted hairs & $3,563.8 \pm 1,070.75$ & $2,824.2 \pm 667.30$ & 5.978 & $<0.001^{\star \star \star}$ \\
\hline Width (mm) & $14.9 \pm 3.29$ & $13.2 \pm 2.16$ & 4.259 & $<0.001^{\star \star \star}$ \\
\hline Length (mm) & $25.8 \pm 3.15$ & $22.4 \pm 1.92$ & 9.318 & $<0.001^{\star \star \star}$ \\
\hline Laxity (mm) & $19.4 \pm 3.72$ & $18.0 \pm 3.04$ & 2.984 & $<0.001^{* *}$ \\
\hline Elasticity (mm) & $7.4 \pm 2.68$ & $7.2 \pm 1.75$ & 0.649 & 0.517 \\
\hline Elasticity/laxity & $38.1 \pm 13.25$ & $40.1 \pm 8.35$ & -1.313 & 0.191 \\
\hline Glidability (mm) & $12.1 \pm 3.68$ & $10.8 \pm 2.49$ & 2.954 & $0.004^{* \star}$ \\
\hline Glidability/laxity & $62.3 \pm 13.68$ & $60.0 \pm 8.35$ & 1.472 & 0.143 \\
\hline \multicolumn{5}{|l|}{ Design width (\%) } \\
\hline Laxity & $77.7 \pm 15.40$ & $74.5 \pm 11.44$ & 1.729 & 0.085 \\
\hline Elasticity & $237.5 \pm 117.66$ & $193.4 \pm 48.30$ & 3.576 & $<0.001^{\star \star \star}$ \\
\hline Glidability & $134.4 \pm 65.69$ & $127.3 \pm 30.57$ & 0.965 & 0.336 \\
\hline \multicolumn{5}{|l|}{ Pain score } \\
\hline POD 1 & $1.8 \pm 1.02$ & $2.3 \pm 1.04$ & -3.193 & $0.002^{* *}$ \\
\hline POD 2 & $1.2 \pm 0.93$ & $1.8 \pm 1.01$ & -3.911 & $<0.001^{\star \star \star}$ \\
\hline POD 3 & $0.7 \pm 0.87$ & $1.2 \pm 1.02$ & -3.554 & $<0.001^{\star * *}$ \\
\hline POD 4 & $0.4 \pm 0.72$ & $0.8 \pm 0.96$ & -2.951 & $0.004^{\star *}$ \\
\hline POD 5 & $0.3 \pm 0.61$ & $0.5 \pm 0.79$ & -1.760 & 0.080 \\
\hline POD 7 & $0.1 \pm 0.39$ & $0.3 \pm 0.66$ & -2.714 & $0.007^{\star *}$ \\
\hline
\end{tabular}


Table 2. Pain score by date (FUT group) $(n=202)$

\begin{tabular}{|c|c|c|c|c|c|c|}
\hline Pain score & POD 1 & POD 2 & POD 3 & POD 4 & POD 5 & POD 7 \\
\hline 0 & $16(7.0)$ & 33 (15.5) & $86(42.0)$ & $128(64.0)$ & $151(74.5)$ & 171 (84.5) \\
\hline 1 & 37 (18.5) & $80(40.0)$ & 67 (33.5) & 45 (22.5) & $35(17.5)$ & $22(11.0)$ \\
\hline 2 & 95 (47.5) & 55 (27.5) & $32(16.0)$ & $19(8.5)$ & $11(5.5)$ & $7(3.5)$ \\
\hline 3 & $35(17.5)$ & 30 (15.0) & $16(8.0)$ & $10(5.0)$ & $5(2.5)$ & $2(1.0)$ \\
\hline 4 & $17(8.5)$ & $4(2.0)$ & $1(0.5)$ & 0 & 0 & 0 \\
\hline 5 & $2(1.0)$ & 0 & 0 & 0 & 0 & 0 \\
\hline
\end{tabular}

Table 3. Difference in postoperative pain (Wong-Baker Scale) by elapsed time after FUT surgery $(n=202)$

\begin{tabular}{|lcccc|}
\hline & Mean & SD & P-value $^{\text {a) }}$ & F-value \\
\hline POD 1 (a) & 2.03 & 1.0505 & $<0.001$ & 133.103 \\
POD 2 (b) & 1.47 & 0.9982 & & \\
POD 3 (c) & 0.91 & 0.9702 & \\
POD 4 (d) & 0.56 & 0.8575 & \\
POD 5 (e) & 0.36 & 0.6995 & \\
POD 7 (f) & 0.21 & 0.5430 & \\
\hline FUT, follicular unit transplantation; POD, postoperative day. \\
a)Statistical significances was tested by one-way analysis of variances among \\
groups. A sign of inequality indicate significant difference between groups based \\
on Scheffe multiple comparison. Post hoc analysis: a $>$ b, c, e, f; b $>c, d, e, f ; b>$ \\
d, e, f; c $>f$ f.
\end{tabular}

nor harvest, or laxity, elasticity, and glidability of the scalp $(\mathrm{P}>0.05)$. The only variable with statistical significance was sex. Postoperative pain was greater in female than male patients; the analysis showed significant differences on postoperative day 1 $(t=-3.193, \mathrm{P}<0.01), 2(\mathrm{t}=-3.911, \mathrm{P}<0.001), 3(\mathrm{t}=-3.554$, $\mathrm{P}<0.001), 4(\mathrm{t}=-2.951, \mathrm{P}<0.01)$, and $7(\mathrm{t}=-2.714, \mathrm{P}<0.01)$ (Fig. 1).

\section{Follicular unit extraction}

Difference in postoperative pain (Wong-Baker Scale) after hair transplantation by FUE according to elapsed time after surgery

Patients experienced the most severe pain on postoperative day 1 with an average of 1.26 points, which decreased to $0.67,0.31$, $0.21,0.18$, and 0.13 points on days $2,3,4,5$, and 7 , respectively.

Post hoc analysis was conducted to identify significant betweengroup differences, and showed that postoperative pain was significantly greater on postoperative day 1 than on days $2,3,4,5$, and 7 and was significantly greater on day 2 than on day 7 (Table 4 ).

\section{Difference in postoperative pain (Wong-Baker Scale) between} male and female patients according to time after surgery

The change in the degree of postoperative was not statistically significant in either male or female patients $(\mathrm{P}>0.05)$.

\section{Fig. 1. Postoperative pain between female and male patients}

Five-point Wong-Baker Faces Pain Scale: 0 point (no hurt) to 5 points (hurts worst).

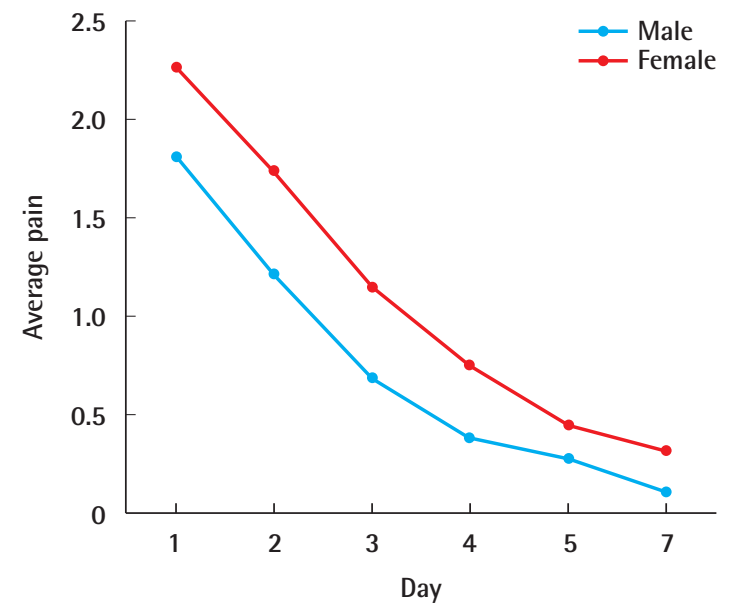

Table 4. Difference in postoperative pain (Wong-Baker Scale) by elapsed time after FUE surgery $(n=39)$

\begin{tabular}{|lccc|}
\hline & Mean & SD & P-value \\
\hline POD 1 (a) & 1.26 & 1.0442 & $<0.001$ \\
POD 2 (b) & 0.67 & 0.8983 & \\
POD 3 (c) & 0.31 & 0.6136 & \\
POD 4 (d) & 0.21 & 0.4690 & \\
POD 5 (e) & 0.18 & 0.5064 & \\
POD 7 (f) & 0.13 & 0.4091 & \\
\hline FUE, follicular unit extraction; POD, postoperative day. & \\
Scheffe test: $a>b, c, d, e, f ; b>f$. & & \\
\end{tabular}

\section{FUT versus FUE}

The independent-sample t-test was used to identify differences in postoperative pain after hair transplantation between the FUT and FUE groups, and the results are shown in Table 4. A statistically significant difference was found between the two methods on days $1,2,3$, and 4 .

On postoperative days $1,2,3$, and 4 , the pain scores were higher in patients who underwent FUT than in those who under- 
Table 5. Comparison of postoperative pain after FUT and FUE

\begin{tabular}{|lccc|}
\hline & FUT $(\boldsymbol{n}=\mathbf{2 0 2})$ & FUE $(\boldsymbol{n}=\mathbf{3 9})$ & P-value \\
\hline POD 1 & $2.03 \pm 1.0505$ & $1.26 \pm 1.0442$ & $<0.001$ \\
POD 2 & $1.47 \pm 0.9982$ & $0.67 \pm 0.8983$ & $<0.001$ \\
POD 3 & $0.91 \pm 0.9702$ & $0.31 \pm 0.6136$ & $<0.001$ \\
POD 4 & $0.56 \pm 0.8575$ & $0.21 \pm 0.4690$ & $<0.001$ \\
POD 5 & $0.36 \pm 0.6995$ & $0.18 \pm 0.5064$ & 0.066 \\
POD 7 & $0.21 \pm 0.5430$ & $0.13 \pm 0.4091$ & 0.385 \\
\hline $\begin{array}{l}\text { Values are presented as mean } \pm \text { SD. } \\
\text { FUT, follicular unit transplantation; FUE, follicular unit extraction; POD, post- } \\
\text { Operative day. }\end{array}$ & \\
\hline
\end{tabular}

went FUE ( 2.03 vs. $1.26,1.47$ vs. $0.67,0.91$ vs. 0.31 , and 0.56 vs. 0.21 , respectively) (Table 5, Fig. 2).

\section{DISCUSSION}

Postoperative pain is one of the most frequent sources of anxiety and fear among patients planning to undergo not only hair transplant surgery, but indeed all types of surgical procedures [7]. Proper management of postoperative pain is essential for humanitarian needs, as well as for reducing nociception-induced responses to surgery, autonomic reflexes adversely affecting organ function, and other undesirable results [8].

One of the most common inquiries from patients during consultations is "Does it hurt?" Patients with extreme surgical fear may be hesitant to undergo hair transplantation, and those who have experienced excruciating pain may not return for subsequent sessions despite the fact that additional transplantation is necessary due to alopecia progression [2]. Therefore, proper management of pain is of great importance.

For patients who undergo hair transplantation, pain mainly occurs during and after surgery. Vibrator application, ice packing, and sedative anesthesia can be used to alleviate the pain induced during surgery. With the help of such methods, patients rarely complain of pain during surgery [9-12].

Postoperative pain following hair transplant surgery mainly arises from the donor area, rather than from the recipient area. Unlike FUE, harvesting by the FUT method involves pulling two edges of an elliptical wound to be sutured, aggravating pain due to the additional stimulus to sensory nerves caused by stretching the skin. Likewise, the FUT method is more painful than the FUE method; it also involves a linear scar, but does not require donor area shaving. In contrast, the FUE method is less painful and does not leave a linear donor scar, but it necessitates shaving the donor area. However, the non-shaven FUE method can be a satisfactory option when shaving is not acceptable. Compared to grafts harvested by FUE, the hair shafts of which
Fig. 2. Postoperative pain by elapsed time after surgery

Five-point Wong-Baker Faces Pain Scale: 0 point (no hurt) to 5 points (hurts worst). FUE, follicular unit extraction; FUT, follicular unit transplantation.

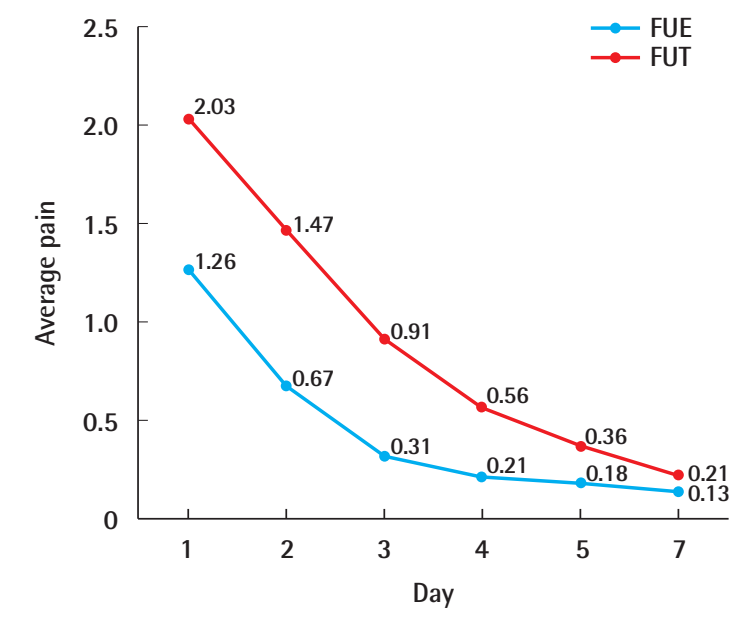

measure approximately $1 \mathrm{~mm}$, those harvested by the FUT method are longer, which allows the surgeon to identify hair curling. Strip harvesting is recommended for cases where identification of hair curling in the process of graft placing is crucial, such as eyebrow restoration surgery or when donor shaving is impossible. The FUE method is suitable for patients with insufficient scalp laxity for FUT or with extreme fear of postoperative pain.

In the present study, postoperative pain after FUT surgery exhibited great variety and was not significantly associated with any of the examined factors. Pain was very subjective. In the FUT group, the average pain score was 2.03, 1.47, 0.91, 0.56, 0.36 , and 0.21 on postoperative day $1,2,3,4,5$, and 7 , respectively. Because the degree of pain decreased to $<1.0$, the postoperative pain can technically be said to have disappeared within 2 days after surgery. In contrast, the pain scores in the FUE group were $1.26,0.67,0.31,0.21,0.18$, and 0.13 on postoperative days $1,2,3,4,5$, and 7 , respectively. No statistically significant difference was found after the 2 nd day, and the degree of pain on the 2 nd day dropped to a value of $<1.0$ (i.e., 0.67 ).

In the comparison of the FUT and FUE methods, postoperative pain was greater in the FUT group than in the FUE group on postoperative day 1 (2.03 and 1.26 points, respectively), day 2 ( 1.47 and 0.67 points), day 3 ( 0.91 and 0.31 points), and day 4 ( 0.56 and 0.21 points). The pain scores were significantly higher in the FUT than FUE group from the first to fourth day postoperatively. The present study has several findings of critical clinical importance. First, postoperative pain after hair transplantation was much greater in the FUT group than in the FUE 
group. Therefore, patients with extreme fear of surgery can be recommended to undergo hair transplantation with the FUE method. Hair surgeons are generally aware of this phenomenon, but no previous report has objectively compared the difference in postoperative pain induced by the two methods. Therefore, the present investigation will provide hair surgeons excellent guidelines for a better understanding of pain characteristics, as well as detailed information about postoperative pain. Second, postoperative pain was almost completely resolved by the 2 nd day. Thus, although pain was measurably greater after FUT surgery than after FUE surgery, the FUT method never induced excruciating pain, and the pain subsided within 2 days. Patients with an occupation that requires extreme concentration or those with anxiety and fear toward postoperative pain can receive an advance explanation that pain usually subsides by day 3 . Third, postoperative pain tended to be very subjective and was not closely related to scalp laxity or the amount of donor harvest. In fact, no statistically significant relationship was found between the various factors examined and postoperative pain. Therefore, the management of postoperative pain after FUT should involve attention and care from clinicians regardless of the degree of scalp laxity. Alopecia is a progressive entity; hair loss may continue even with medication, and the existing hair may thin over time. Without proper medication adherence, the process of hair loss can be accelerated as well. Hence, a single hair transplant session is not a cure for alopecia. There is always a possibility of the need for second or third surgery sessions. Therefore, it is important for the patient to maintain a positive perception of the surgical procedure so that they do not avoid further hair transplantation because of having experienced excruciating postoperative pain. In such cases, however, harvesting by the FUE method can be an excellent option for subsequent hair transplantation.

The present report on postoperative pain provides indisputable evidence for patient education regarding the postoperative course and strong grounds for future studies on further amelioration of postoperative pain, benefitting both patients and practitioners.

\section{NOTES}

\section{Conflict of interest}

No potential conflict of interest relevant to this article was reported.

\section{Ethical approval}

The study was approved by the Institutional Review Board of the Korea National Institute of Bioethics Policy (IRB No. 2015-
0144-001) and performed in accordance with the principles of the Declaration of Helsinki. Written informed consents were obtained.

\section{Author contribution}

Conceptualization, methodology: Park JH. Writing - original draft: Park JH, Kim YS. Writing - review \& editing: Park JH, Na YC.

\section{ORCID}

Yang Seok Kim https://orcid.org/0000-0003-3161-7284

Young Cheon Na https://orcid.org/0000-0002-3479-3243

Jae Hyun Park https://orcid.org/0000-0002-9230-4102

\section{REFERENCES}

1. Apfelbaum JL, Chen C, Mehta SS, et al. Postoperative pain experience: results from a national survey suggest postoperative pain continues to be undermanaged. Anesth Analg 2003;97:534-40.

2. Nusbaum BP. Techniques to reduce pain associated with hair transplantation: optimizing anesthesia and analgesia. Am J Clin Dermatol 2004;5:9-15.

3. Nussbaum BP. Reducing pain during hair transplant lidocaine infiltration. Dermatol Surg 2001;27:98.

4. Bernstein RM, Rassman WR. Limiting epinephrine in large hair transplant sessions. Hair Transplant Forum Int 2000;10: 39-41.

5. Park JH. Association between scalp laxity, elasticity, and glidability and donor strip scar width in hair transplantation and a new elasticity measuring method. Dermatol Surg 2017; 43:574-81.

6. Garra G, Singer AJ, Domingo A, et al. The Wong-Baker pain FACES scale measures pain, not fear. Pediatr Emerg Care 2013;29:17-20.

7. Melzack R, Wall PD. Pain mechanisms: a new theory. Science 1965;150:971-9.

8. Kehlet H, Dahl JB. The value of "multimodal" or "balanced analgesia" in postoperative pain treatment. Anesth Analg 1993;77:1048-56.

9. Fosko SW, Gibney MD, Harrison B. Repetitive pinching of the skin during lidocaine infiltration reduces patient discomfort. J Am Acad Dermatol 1998;39:74-8.

10. Parsley WM. Vibrators. Hair Transplant Forum Int 2000;10: 20.

11. Barusco MN, Leavitt HL, Kirk R. The use of a computerized anesthesia injection system to minimize pain during hair transplant surgery. Hair Transplant Forum Int 2001;4:107- 
9.

12. True RH, Elliott RM. Microprocessor-controlled local anes- thesia versus the conventional syringe technique in hair transplantation. Dermatol Surg 2002;28:463-8. 